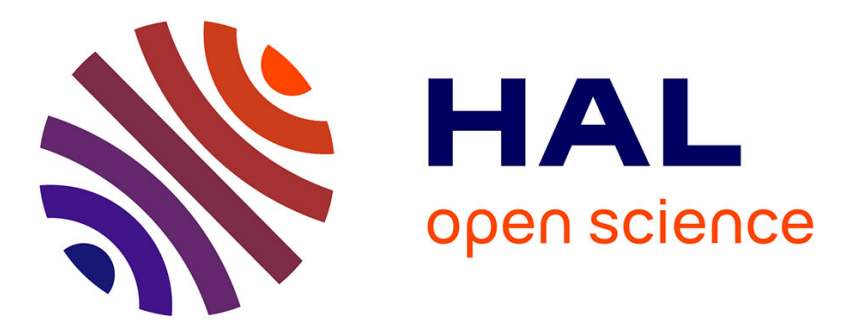

\title{
Understanding frothing of liquid mixtures: A surfactant-like effect at the origin of enhanced liquid film lifetimes
}

H.-P Tran, M Arangalage, L Jørgensen, N Passade-Boupat, F Lequeux, L Talini

\section{To cite this version:}

H.-P Tran, M Arangalage, L Jørgensen, N Passade-Boupat, F Lequeux, et al.. Understanding frothing of liquid mixtures: A surfactant-like effect at the origin of enhanced liquid film lifetimes. Physical Review Letters, 2020, 125, pp.178002. hal-02939747

\section{HAL Id: hal-02939747 https://hal.science/hal-02939747}

Submitted on 15 Sep 2020

HAL is a multi-disciplinary open access archive for the deposit and dissemination of scientific research documents, whether they are published or not. The documents may come from teaching and research institutions in France or abroad, or from public or private research centers.
L'archive ouverte pluridisciplinaire HAL, est destinée au dépôt et à la diffusion de documents scientifiques de niveau recherche, publiés ou non, émanant des établissements d'enseignement et de recherche français ou étrangers, des laboratoires publics ou privés. 


\title{
Understanding frothing of liquid mixtures:
}

\section{A surfactant-like effect at the origin of enhanced liquid film lifetimes}

\author{
H.-P. Tran ${ }^{1,2}$, M. Arangalage ${ }^{1,2}$, L. Jørgensen ${ }^{1,2}$, N. Passade-Boupat ${ }^{2,3}$, F. Lequeux ${ }^{1,2}$ and L. Talini ${ }^{1,2,4}$ \\ ${ }^{1}$ CNRS, Sciences et Ingénierie de la Matière Molle, ESPCI Paris, PSL Research University, Sorbonne Université, 75005 \\ Paris, France. \\ 2 Laboratoire Physico-Chimie des Interfaces Complexes, ESPCI, 10 rue Vauquelin, 75005 Paris, Bâtiment CHEMSTARTUP, \\ Route Départementale 817, 64170 Lacq, France. \\ 3 Total S.A. 64170 Lacq, France. \\ ${ }^{4}$ Present address : CNRS, Surface du Verre et Interfaces, Saint-Gobain, 93300 Aubervilliers, France.
}

Abstract: The formation of froth in mixtures of liquids is well documented, in particular in oil mixtures. However, in non-volatile liquids and in the absence of surface-active molecules, the effect increasing liquid film lifetimes had not been identified. We suggest a stabilizing mechanism resulting from the non-linear variations of the surface tension of a liquid mixture with its composition. We report experimental lifetimes of froths in binary mixtures and we show their variations are well predicted by the suggested mechanism. We demonstrate it prescribes the thickness reached by films before their slow drainage, thickness which correlates well with froth lifetimes for both polar and non-polar liquids.

Foams are metastable dispersions of gas in liquids. In the past decades, the processes at the origin of their finite lifetimes have been extensively studied in surfactant solutions [2]. Among the destabilizing mechanisms, one results from the capillary drainage of liquid films in the Plateau borders where three liquid films meet. The pressure drop associated with the curved gas/liquid interfaces at the Plateau borders induces a capillary suction of the liquid, leading to film thinning. In presence of surfactants adsorbed at the interfaces, thinning can be opposed by a repulsion between both film interfaces, either of steric or charge-induced nature. In addition, film rupture can be delayed by the surface tension gradient originating from local extension of the film surface. A local decrease in surfactant concentration generates an increase in surface tension, driving a Marangoni flow opposing drainage [3]. A measurement of that effect is the Gibbs elasticity, which associated modulus relates the excess surface tension to the relative increase of surface area $[4,5]$. However, the influence of Gibbs elasticity on foam stability is still an open question [6].

Marangoni flows are also invoked to explain the large influence of contaminants on the stability of liquid films, even in the absence of purposely added surfactants [7]. However, in pure liquids of low surface tensions such as oils, contaminant-induced effects are small and the lifetime of films is so short that generally no foaming is observed in pure non-polar 
liquids. In contrast, mixtures of liquids have been shown to form froths, i.e. poorly stable foams, even with non-polar liquids, as first evidenced decades ago [8]. Using binary and ternary mixtures, froth lifetimes up to a few tens of seconds were reported, that increased in the vicinity of critical points. As in pure liquids, the net interaction between interfaces of a liquid film are attractive owing to van der Waals forces. Since they are also free of surfactants, a new stabilizing mechanism has to be invoked in these liquids. In order to clearly distinguish the mechanism of thin films stabilisation from the effect of disjoining pressure, we will call the phenomenon frothing instead of foaming. The frothing behaviour was attributed to the variations of surface activity occurring before phase separation but was not quantitatively described. Similar findings were reported in the literature for mixtures of partially miscible liquids $[9,10]$. One of the salient features is the existence of a sharp maximum of foam lifetime at a given mixture composition.

More recently, asymmetric evaporation in liquid mixtures has been pointed out to be responsible for the stabilization of foams in some oil mixtures [11]. When the component with the smaller surface tension is the more volatile, its evaporation results in a surface tension gradient generating a Marangoni flow that stabilizes a liquid film. In contrast, when the more volatile component has the larger surface tension, the Marangoni effect is expected to destabilize films.

In the present work, we report frothing experiments with mixtures of fully miscible liquids that are either polar or non-polar. In agreement with past results, the lifetimes of the formed foams are small (a few tens of seconds) and exhibit a maximum with compositions of mixtures. Stabilization does not result from evaporation-induced Marangoni effects since evaporation has a destabilizing effect in most mixtures. In addition, in this peculiar systems in which surface and bulk are of close compositions with extremely fast exchanges between them, there is no effect of interfacial viscosity. In contrast, we show the experimental data can be quantitatively described by introducing a thickness-dependent surface tension of the liquid mixture, resulting from concentration of the species with the lower surface tension at the interface with air. Within this context, we will show that the only condition for frothing consists in non-linear variations of surface tensions of mixtures with their compositions.

We have investigated the frothing behaviour of binary mixtures respectively of toluene and linear alkanes and of cyclopentanol and linear alcohols. An additional mixture of linear alcohols was tested. All liquids were high purity ( $>99 \%)$ ones supplied by Sigma Aldrich. The linear species were of different carbon chain lengths: from heptane to decane for the alkanes 
and from pentanol to heptanol for the alcohols. The surface tension of alkanes (respectively alcohols) increases with the length of the carbon chain, but is smaller than the surface tension of toluene (respectively cyclopentanol) in the investigated range [12]. The surface tensions of all liquids were systematically measured with a Teclis rising bubble tensiometer. In the following, the surface tension of a mixture is denoted as $\gamma ; \gamma_{i}(i=1,2)$ is the surface tension of pure liquid $i$, and $x_{i}$ its molar fraction in the mixture. Liquid 1 refers to the species with the lowest surface tension, i.e. either the linear alkane or linear alcohol. The surface tension difference is $\Delta \gamma=\gamma_{2}-\gamma_{1}$ and $\Delta \gamma>0$.

For all alkane/toluene and alcohol/cyclopentanol mixtures the surface tension was found to exhibit a sub-linear variation [13], as the one for decane/toluene shown in Fig. 1. It suggests the existence of a surface adsorption layer for the species with the lower surface tension, such as the one evidenced both numerically [14] and experimentally [15] in water-alcohol mixtures. We show in the following that it is at the origin of the enhanced lifetimes of films of liquid mixtures. In contrast the surface tension of alkane mixtures (such as the one in Fig. 1) varies linearly with composition indicating identical bulk and surface compositions.

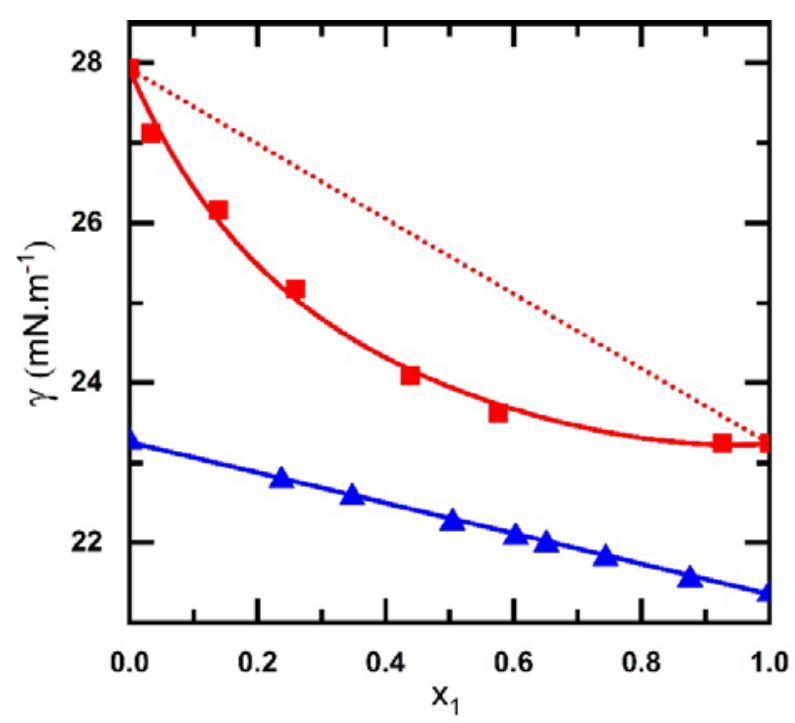

Fig. 1: Surface tensions of decane/toluene (red) and octane/decane (blue) as a function of the molar fraction of the species with the lowest surface tension (respectively decane and octane). The full lines are guides to the eye.

Surface tension variations can be described using available models for binary mixtures. A simple approximation considers linear variation of surface tension with surface molar concentrations of each species $\Gamma_{i}(\mathrm{i}=1,2)[16]$ :

$$
\gamma \approx \Gamma_{1} \sigma_{1} \gamma_{1}+\Gamma_{2} \sigma_{2} \gamma_{2}
$$


where $\sigma_{i}$ is the molar area of species $i$.

Writing $\Gamma_{2} \sigma_{2}=1-\Gamma_{1} \sigma_{1}$ and replacing it in eq. (1) provides a relation between $\gamma$ and $\Gamma_{1}$. Surface concentrations can thus be inferred from the surface tension values of the binary mixture. In the case of a linear variation of the interfacial tension with bulk composition, the surface population is the same as the bulk one, i.e. $\Gamma_{i} \sigma_{i}=x_{i}$. In the following we show that case corresponds to non-foaming mixtures.

More elaborate models for surface tensions of mixtures are available; we have tested one in which molecular interactions are accounted for by a Flory parameter [17] and found it equivalently described our frothing data. Therefore, our analysis is not model-dependent and in the following we use the simple approximation of eq. (1).

Foaming experiments were performed with Bikerman columns [18, 19], i.e. glass columns of radius $R=1 \mathrm{~cm}$ and height $L=30 \mathrm{~cm}$ with a porous filter at their bottom (Robu, porosity: $10-16 \mu \mathrm{m})$. The columns were filled with liquids up to a height $H_{0}=10 \mathrm{~cm}$ and were part of a closed air circuit, in which air was continuously pumped and injected through the porous at flow rate $Q=6 \mathrm{~mL} \cdot \mathrm{s}^{-1}$ [20]. The stationary froth height $H$ reached was measured from video images. The froth lifetime, corresponding to the time during which bubbles are convected before bursting, is defined as $\tau=H \pi R^{2} / Q$. We have checked that it does not depend on initial height $H_{0}$ and flow rate $Q$ in the probed ranges. Since capillary drainage is the destabilizing mechanism of the studied froths, time $\tau$ is expected to linearly vary with liquid viscosity $\mu[21,22]$. Mixture viscosities were either measured with a rheometer (Low Shear 400, Lamy rheology) or computed using the empirical Kendall-Monroe equation [23], shown to be adequate for alkane/toluene mixtures [24]. In the following, we report values of the ratio $\tau / \mu$, which provides a measurement of foamability that is independent of both the injection conditions and liquid viscosity.

Figure 2 shows the ratio $\tau / \mu$ as a function of the alkane molar fraction $x_{1}$ for the different alkane/toluene mixtures. In agreement with past results [8], the variations of both time $\tau$ and foamability $\tau / \mu$, are non-monotonic, whereas the viscosity varies monotonically with composition. A maximum for $\tau / \mu$ is reached at an alkane fraction slightly depending on the length of the carbon chain of the alkane. The maximum value of the foamability more strongly depends on the alkane: it is for instance about 4 times larger for decane than for heptane. Similar features were found with alcohol/cyclopentanol mixtures [25]. 


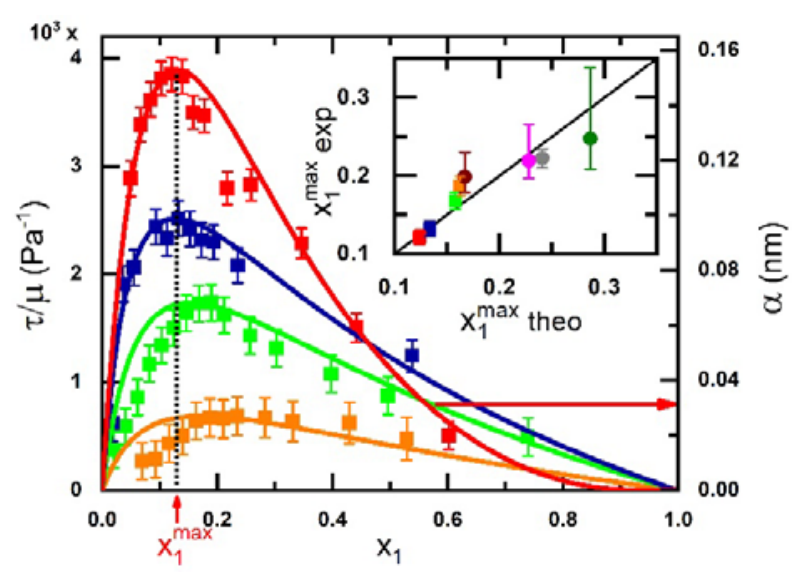

Fig. 2: Experimental foamability (squares) defined as the ratio of foam lifetimes and liquid viscosities (left axis) as a function of alkane molar fraction for mixtures of toluene with decane (red), nonane (blue), octane (green) and heptane (orange). Error bars correspond to uncertainties on measured foam heights. The length $\alpha$ characterizing the relative surface tension variation with film thickness (eq. (5)) is shown in full line. Scale of right axis is for the decane/toluene mixture only, other $\alpha$-curves are in arbitrary unit. Inset: Molar fraction for which the foamability was measured to be maximum as a function of its value predicted by eq. (4). Data for alkane/toluene (squares) and alcohol/cyclopentanol mixtures (circles, same as in Fig. 4). Horizontal error bars are too small to appear.

We attribute the frothing behaviour of mixtures to the role of the different species at the interface: the non-linear variations of surface tension of mixtures with linear alkane or alcohol fraction indicates that the surface concentration of respectively alkane or alcohol is larger than its bulk one. It suggests the existence of a surface adsorption layer of molecular thickness such as the one in water/alcohol mixtures $[14,15]$. The ability to quickly pump molecules from the bulk to that layer results in a thickness-dependent surface tension, which is at the origin of the enhanced stability of thin films in liquid mixtures.

More precisely, we consider a liquid film of thickness $h$ and surface $S$. Thickness $h$ being much smaller than the other dimensions, all processes occurring along the $h$ direction can be considered as instantaneous compared to the ones in lateral directions. In particular, the characteristic molecular diffusion time through the film thickness $h^{2} / D \approx 1 m s$ (with $D$ the diffusion coefficient and $h=1 \mu \mathrm{m}$ ), is small compared to the timescales of the processes we consider. Consequently, we assume that thermodynamical equilibrium between bulk and surfaces of the film is reached instantaneously. 
Within the foam, films are connected to menisci called Plateau borders in which the capillary pressure drop induces a suction (see fig. 3). As liquid is drawn off the film, two stages occurring at very different timescales can be considered. At a first stage, surface tension is uniform and an extensional flow is established, as in liquid films with mobile interfaces in which no pinching occurs [26]. The film is stretched and gets thinner at almost constant volume, and, at one point, its surface concentration in the species with the lowest surface tension must decrease because it surface-to-volume ratio has increased. Surface tension thus increases in the film allowing equilibrium of tensions to be reached. From that instant, thinning is further associated with surface tension gradient, resulting in a zero-velocity (solidlike) condition at the interfaces [27]. The flow is therefore a much slower Poiseuille flow and the film is further subjected to marginal pinching [28]. No full theoretical description is available for the latter process, and it is out of the scope of the present work. However, since the first stretching stage is short, it is the slow drainage stage that sets film lifetime, which therefore depends on the initial thickness. The latter, we denote $h_{f}$, is the one reached at the end of first stage, when the increase of surface tension in the film exactly compensates capillary suction and tension in the meniscus. Considering the equilibrium reached at that point allows the determination of $h_{f}$. Our analysis is based on the assumption that the film lifetime correlates with the value of $h_{f}$.

As schematized in Fig. 3, the film of thickness $h_{f}$ is connected to a Plateau border. Pressure equilibrium is not reached but equilibrium of the tensions in the film is nevertheless satisfied, yielding at any $z$ :

$$
2 \gamma(h) \cos [\theta(z)]+\Delta P(h) h(z)=2 \gamma\left(h_{f}\right)
$$

Where $\theta(z)$ is the local angle of the film with the $z$-axis direction and $h(z)$ the local film thickness. $\Delta P(h)=\gamma(h) d^{2}(h / 2) / d z^{2}$ is the Laplace pressure difference between the gas and the liquid in the meniscus written in the thin-film approximation with first-order terms only. The right-hand term of eq. (2) is the left-hand term written in the flat part of the film for which $\theta=0$ and $\Delta P(h)=0$. Equation (2) is similar to the one for film equilibrium with significant disjoining pressure [29]. Here, disjoining pressure is negligible since $h_{f}$ is assumed to be large, and equilibrium is reached because surface tension in the flat film is larger than in the Plateau border. 


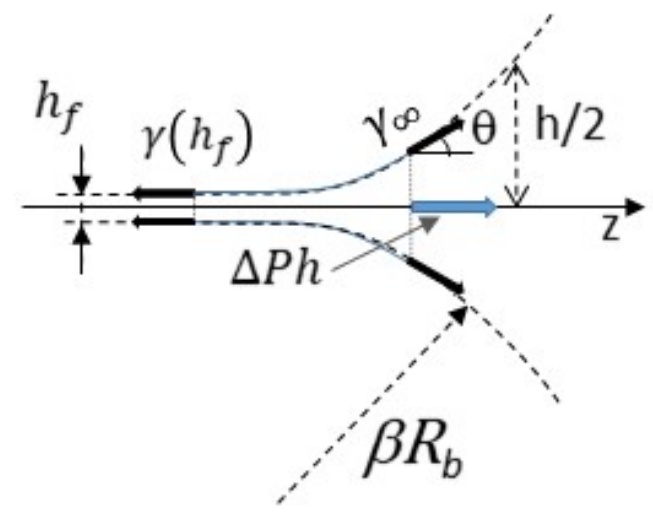

Fig. 3: Schematic of a liquid film of thickness $h_{f}$ connected to a Plateau border in the foam and the forces (per unit length) it is submitted to.

We now show that the thickness-dependent surface tension $\gamma(h)$ can be derived from the variations of surface tension with composition. We assume the film, of total surface $S$, is formed from a volume $V_{0}$ of mixture, of initial molar fractions $x_{i}^{0}$ in species of respective molar volumes $v_{i}(i=1,2)$, and of equilibrium surface tension $\gamma_{\infty}=\gamma\left(x_{i}^{0}\right)$. As the film thins down and its surface to volume ratio $S / V$ increases, its bulk molar fractions $x_{i}$ vary. A geometrical relation between $x_{i}$ and $x_{i}^{0}$ can be derived by combining conservation of molecules, $\Gamma_{i} S+x_{i} V / v_{i}=x_{i}^{0} V_{0} / v_{i}$ and constant volume and surface densities $\sum_{i} x_{i}^{0}=$ $\sum_{i} x_{i}=\sum_{i} \Gamma_{i} \sigma_{i}=1$ :

$$
x_{1}-x_{1}^{0}=\frac{S\left(v_{1} \Gamma_{2} \sigma_{2}\left(-1+x_{1}^{0}\right)+v_{2}\left(1-\Gamma_{1} \sigma_{1}\right) x_{1}^{0}\right)}{S v_{2}\left(-1+\Gamma_{1} \sigma_{1}\right)+\left(V_{0}-S v_{1} \Gamma_{1}\right) \sigma_{2}}
$$

Further expanding that relation in powers of $S$ and using the linearized relation $\gamma(h)-\gamma_{\infty}=$ $\left(x_{i}-x_{i}^{0}\right)\left(\partial \gamma / \partial x_{1}\right)_{x_{1}=x_{1}^{0}}$, we obtain the asymptotical limit of surface tension variation. Replacing surface concentrations by their expressions inferred from eq. (1), and replacing $S$ by $V_{0} / h$, finally yields :

$$
\gamma(h)-\gamma_{\infty}=\frac{2}{h}\left(\frac{\partial \gamma}{\partial x_{1}}\right)_{x_{1}=x_{1}^{0}}\left(\frac{\frac{v_{1}}{\sigma_{1}}\left(\gamma_{\infty}-\gamma_{2}\right)\left(1-x_{1}^{0}\right)+\frac{v_{2}}{\sigma_{2}}\left(\gamma_{\infty}-\gamma_{1}\right) x_{1}^{0}}{\gamma_{2}-\gamma_{1}}\right)+O\left(\frac{1}{h^{2}}\right)
$$

Equation (4) is valid in the limit $h \gg v_{i} / \sigma_{i}$ (species $i$ being the one with the largest molecules), corresponding to thicknesses much larger than the molecular size. In this range, 
the second-order term of the expansion is smaller than the first-order one and $\gamma(h)$ remains larger than $\gamma_{\infty}$.

According to eq. (4), the effect stabilizing liquid films vanishes for finite thicknesses provided surface tension varies linearly with composition, i.e. $\gamma_{\infty}=\gamma_{1} x_{1}^{0}+\gamma_{2}\left(1-x_{1}^{0}\right)$. Non-linear variations of surface tensions are therefore at the origin of the thickness-dependent surface tension.

In the following, we write eq. (4) under the form:

$$
\gamma(h)=\gamma_{\infty}(1+\alpha / h)
$$

where $\alpha$ is a characteristic length that can be computed from molar volumes and surfaces of the two liquids and from the derivative of $\gamma\left(x_{1}\right)$. Calculation of the latter is performed with empirical functions fitting the experimental data (full line in fig. 1). We have found $\alpha$ ranges from $10^{-2}$ to $10^{-1} \mathrm{~nm}$, which corresponds to surface tension variations of a few $\mu \mathrm{N} . \mathrm{m}^{-1}$ for a $1 \mu \mathrm{m}$-thick film. The increase of surface tension is therefore very small but large enough to induce Marangoni effects.

In light of the previous analysis, the experimental variations of foamability with mixture composition can be compared to the ones of $\alpha$, and they are in excellent agreement, as shown in fig. 2. The values of $\alpha$ correspond to the decane/toluene mixture only, other $\alpha$ curves being shown in arbitrary units to superimpose with foamability, with which the relation is not linear (see what follows). A similar agreement was found for polar mixtures [8], and in particular, the composition for maximum foamability is quantitatively predicted (inset to Fig. 2). In addition, no froth could be observed in alkane mixtures. As detailed above, these mixtures exhibit quasi-linear variations of their surface tensions and, according to eq. (4), vanishing film lifetimes are expected, consistently with observations.

Furthermore, the thickness $h_{f}$ reached by the film before it significantly drains, can be determined using eq. (2). Injecting the expression for $\gamma(h)$ yields a differential equation satisfied by $h(z)$ at equilibrium. The equation can be solved numerically and we have found that, in the meniscus, the asymptotical solution to that equation is of parabolic form: $h(z) \cong$ $2 h_{f}+z^{2} \alpha / h_{f}^{2}$ provided $\alpha / h_{f} \ll 1$ [30]. In foams, the meniscus corresponds to a Plateau border which curvature radius can be written as $\beta R_{b}$, where $R_{b}$ is the bubble radius and $\beta$ a numerical prefactor that depends on the liquid volume fraction of the foam, $\varphi_{l}$ following 
$\beta=\sqrt{\varphi_{l} / 0.33}$ [31]. Within this framework, a simple relation is obtained for thickness $h_{f}$ reached by the liquid films before their slow drainage:

$$
h_{f} \cong \sqrt{\alpha \beta R_{b}}
$$

In addition, an estimate of the liquid volume fraction of the foam can be obtained by writing the drainage equation of foams in a stationary state [31]. Assuming the foam is a packing of bubbles which permeability is given by the Kozeny-Carman equation, we find $\varphi_{l} \approx 0.1$ for all mixtures, yielding $\beta=0.55$.

Thickness $h_{f}$ can thus be computed for all mixtures from eq. (6) and in Fig. 4 it is compared to the experimental foamability, made dimensionless by multiplying it by the Laplace pressure difference. Despite the strong approximation of eq. (1), foamability and thickness $h_{f}$ correlate and fig. 4 clearly evidences the existence of a master curve, onto which the data falls for both polar and non-polar liquids in which frothing is observed. In the case of alkane mixtures, thicknesses $h_{f}$ computed from the quasi-linear variations of surface tension with composition are shown by arrows. In these mixtures, no froth height could be measured and consistently, the found values of $h_{f}$ are the smallest of all mixtures.

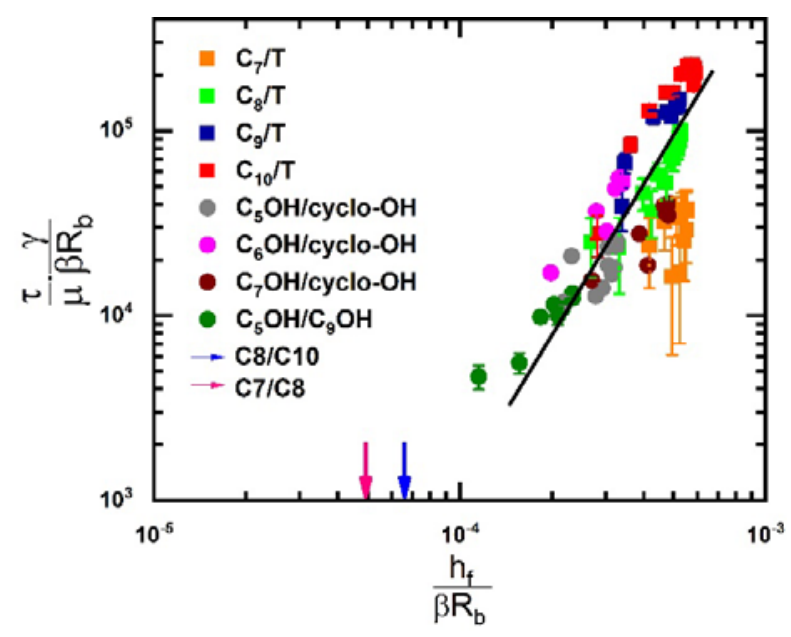

Fig. 4: Dimensionless foamabilities of alkane/toluene and alcohol/cyclopentanol mixtures as a function of the ratio of the predicted film thickness computed from eq. (6) and the radius of curvature of the Plateau border, $\beta R_{b}$. The full line is a guide for the eye. Bubble radius $2 R_{b}=1.6 \mathrm{~mm} \pm 0.3 \mathrm{~mm}$ was measured to be independent of the nature and composition of the mixture, as well as $\beta=0.55$. Arrows indicate the thickness values predicted in mixtures in which no foam could be observed. 
Finally, we emphasize $h_{f} \approx 100 \mathrm{~nm}$, corresponding to negligible van der Waals attraction. It demonstrates that, consistently, the stabilizing effect we depict is of larger range than van der Waals forces.

In conclusion, we have shown frothing of liquid mixtures is explained by a surfactant-like behaviour of the liquid of smaller surface tension. A thickness-dependent surface tension of the mixture results from the non-linear variations of surface tension with composition and it is the resulting thickness-dependent surface tension of liquid films that quantifies the foamability of the mixture. Within this framework, the thickness reached by the liquid films before drainage can be computed from the experimental variations of surface tension with composition. As expected, this thickness and the foamability correlate for liquids of different polarities. It demonstrates the universal nature of the effect, which can have large consequences on processes in which mixtures of liquids are in contact with gases, such as transport of oil in pipes or food processing.

\section{References}

1. Saint-Jalmes, A., Physical chemistry in foam drainage and coarsening. Soft Matter, 2006. 2(10): p. 836-849.

2. Petkova, B., et al., Foamability of aqueous solutions: Role of surfactant type and concentration. Advances in colloid and interface science, 2019. 276: p. 102084-102084.

3. Couder, Y., J.M. Chomaz, and M. Rabaud, ON THE HYDRODYNAMICS OF SOAP FILMS. Physica D, 1989. 37(1-3): p. 384-405.

4. Prins, A., C. Arcuri, and Vandente.M, Elasticity of thin liquid films. Journal of Colloid and Interface Science, 1967. 24(1): p. 84-\&.

5. Vandente.M, J. Lucassen, and Lucassen.Eh, Application of surface thermodynamics to gibbs elasticity. Journal of Physical Chemistry, 1965. 69(6): p. 1798-\&.

6. Stoyanov, S.D. and N.D. Denkov, Role of surface diffusion for the drainage and hydrodynamic stability of thin liquid films. Langmuir, 2001. 17(4): p. 1150-1156.

7. Lhuissier, H. and E. Villermaux, Bursting bubble aerosols. Journal of Fluid Mechanics, 2012. 696: p. 5-44.

8. Ross, S. and G. Nishioka, Foaminess of binary and ternary solutions. Journal of Physical Chemistry, 1975. 79(15): p. 1561-1565.

9. Gracia, J., et al., Transient foaminess, aggregate formation, and wetting behavior in waterphenol mixtures. Journal of Physical Chemistry, 1986. 90(7): p. 1350-1353.

10. Tuinier, R., et al., Transient foaming behavior of aqueous alcohol solutions as related to their dilational surface properties. Journal of Colloid and Interface Science, 1996. 179(2): p. 327 334.

11. Suja, V.C., et al., Evaporation-induced foam stabilization in lubricating oils. Proceedings of the National Academy of Sciences of the United States of America, 2018. 115(31): p. 7919-7924.

12. See Supplemental Material at [URL will be inserted by publisher] for properties of pure liquids.

13. See Supplemental Material at [URL will be inserted by publisher] for curves. 
14. Matsumoto, M., Y. Takaoka, and Y. Kataoka, Liquid vapor interface of water-methanol mixture .1. Computer-simulation. Journal of Chemical Physics, 1993. 98(2): p. 1464-1472.

15. Chen, H., et al., Determination of structure and energetics for Gibbs surface adsorption layers of binary liquid mixture 2. Methanol plus water. Journal of Physical Chemistry B, 2005. 109(16): p. 8064-8075.

16. Eberhart, J.G., The Surface Tension of Binary Liquid Mixtures. J. Phys. Chem, 1966. 70(4): p. 1183-1186.

17. Eriksson, J.C., On the thermodynamics of surface systems. Advances in Chemical Physics, 1964. 6: p. 145-174.

18. Bikerman, J.J., Methods of Measuring Foaminess In Foams: Theory and Industrial Applications. 1953, New-York: Reinhold.

19. Bikerman, J.J., Measurement of Foaminess In Foams. 1973, New York: Springer-Verlag.

20. see supplemental material at [url will be inserted by publisher] for a video of an experiment.

21. Brady, A.P. and S. Ross, The measurement of foam stability. Journal of the American Chemical Society, 1944. 66: p. 1348-1356.

22. Verbist, G., D. Weaire, and A.M. Kraynik, The foam drainage equation. Journal of PhysicsCondensed Matter, 1996. 8(21): p. 3715-3731.

23. Kendall, J. and K.P. Monroe, The viscosity of liquids. II. The viscosity-composition curve for ideal liquid mixtures. Journal of the American Chemical Society, 1917. 39: p. 1787-1802.

24. Iloukhani, H., M. Rezaei-Sameti, and J. Basiri-Parsa, Excess molar volumes and dynamic viscosities for binary mixtures of toluene plus n-alkanes (C-5-C-10) at $T=298.15 \mathrm{~K}$ Comparison with Prigogine-Flory-Patterson theory. Journal of Chemical Thermodynamics, 2006. 38(8): p. 975-982.

25. See Supplemental Material at [URL will be inserted by publisher] for curves.

26. Howell, P.D. and H.A. Stone, On the absence of marginal pinching in thin free films. European Journal of Applied Mathematics, 2005. 16: p. 569-582.

27. Koehler, S.A., et al., Drainage of single Plateau borders: Direct observation of rigid and mobile interfaces. Physical Review E, 2002. 66(4).

28. Aradian, A., E. Raphael, and P.G. de Gennes, "Marginal pinching" in soap films. Europhysics Letters, 2001. 55(6): p. 834-840.

29. Toshev, B.V. and I.B. Ivanov, THERMODYNAMICS OF THIN LIQUID-FILMS .1. BASIC RELATIONS AND CONDITIONS OF EQUILIBRIUM. Colloid and Polymer Science, 1975. 253(7): p. 558-565.

30. See Supplemental Material at [URL will be inserted by publisher] for details.

31. Cantat, I.C.-A., S.; Elias, F.; Graner, F.; Höhler, R.; Pitois, O.; Rouyer, F.; Saint-Jalmes, A., Foams. Structure and Dynamics. 2013: Oxford University Press. 